\title{
Renormalization and fractals among sets beyond (empty) boundaries
}

\author{
Shun Adachi
}

\author{
November 4, 2019
}
Department of Microbiology, Kansai Medical University, 2-5-1 Shin-machi, Hirakata, Osaka 573-1010, JAPAN;
E-mail: f.peregrinusns@mbox.kyoto-inet.or.jp;
ORCID: 0000-0001-7555-8373.

\begin{abstract}
In previous studies, the authors utilized a single-dimensional operationalization of species density that implies induction of hierarchy and time with certain topologies. For further clarification of induced fractals including the relation to renormalization in physics, here a theoretical development is proposed based on a newly identified fact, namely that scaling parameters for magnetization exactly correspond to imaginary parts of Riemann zeta nontrivial zeros. An analogy to magnetization and accompanying Fake Monster Algebra is invoked to lend support to this theory, along with empirical species density data for a wild Dictyostelia community. A master torus and a La-grangian/ Hamiltonian are derived expressing fractal structures as a solution for diminishing divergent terms in renormalization.
\end{abstract}

Keywords: topology; fractal; renormalization; species

\section{Introduction}

In physics, renormalization is a widely applied to accommodate the divergence of terms (e.g., [Benfatto and Gallavotti, 1995, Cardy, 1996]) despite its fragility in rigorous mathematics. Importantly, renormalization could be intrinsically related to the development of fractals, which apparently disappear from the layer of interest, while at a larger scale higher-order fractals are 
manifested. Herein, we commence by deciphering a model of magnetization in a scaling hypothesis by focusing on the fact that the parameters involved are exactly equivalent to the imaginary parts of particular nontrivial zero points of a Riemann zeta function. Utilizing an analogy of magnetization to species dynamics in biology [Adachi, 2019a], we posit that fractals can explain many salient aspects. To approach this problem, we introduce two mathematical ideas - étale and Frobenioid.

Broadly speaking, an étale is a category-theoretic abstraction of the notion of a category if it has a lifting property that is analogous to being a local diffeomorphism, i.e., intuitively a function between smooth manifolds that preserves the local differentiable structure. A Frobenioid is a categorytheoretic abstraction of the notion of a category of line bundles or monoids of divisors over a base category of topological localizations such as a Galois [Mochizuki, 2019a]. A Gaussian monoid would roughly correspond to a harmonic function related to an étale or Frobenioid. Thus, $p$ (persistent homology) or $l$ (étale cohomology) in [Adachi, 2019a, Adachi, 2019b] can be evaluated as the number of Gaussian monoids. For Cartesian coordinates (implying entanglement of each line as in [Witten, 2016]), the system according to étale and Frobenioid is described as de Rham side or Hodge filtration, designated as the Frobenius picture in [Mochizuki, 2019b]. For polar coordinates, it is described as the étale side or Galois action on torsion points, designated as the étale picture in [Mochizuki, 2019b]. The Frobenius picture corresponds to Fig. 5 in [Adachi, 2019a]. For the étale picture, a gradient is étale acting on self-organization, whilst a rotation acting on circulation is Frobenioid, both of them acting on divergence. The logarithm of a multiplicative étale picture is an additive Frobenius picture in the sense of complex metrics.

Our biological model in [Adachi, 2019a, Adachi, 2019b] already exhibits properties for real étale, whilst the Frobenioid is an imaginary part of a newly defined complex metric $s$. Introducing automorphic forms and tori would lead to a master Lagrangian inspired by an analogy to the standard model in physics, and further clarifications explain how fractals in a higherorder hierarchy can explain neglecting divergence in the current layer and investing the property to fractals. Through a biological species model that obviously possesses the property for a multilevel hierarchy, herein we propose a toy hybrid model drawing on mathematics, physics, and biology to tackle the divergent terms of interest. 


\section{Field Research \& Experimental Design}

For calculation purposes, Microsoft Excel 16.16.14, GNU Octave 4.0.3, and SageMath 8.8 are used. Data concerning the number of individuals in each population and species were obtained from natural (nonlaboratory) environments. The sampling method is described in [Adachi, 2015, Adachi, 2019a, Adachi, 2019b]. Field experiments were approved by the Ministry of the Environment (Japan), Ministry of Agriculture, Forestry and Fisheries (Japan), Shizuoka Prefecture (Japan), and Washidu Shrine (Japan). The approval numbers are 23Ikan24, 24Ikan72-32, and 24Ikan72-57.

Soil samples were obtained from two point quadrats in the Washidu region of Izu in Japan. The number of individual cellular slime molds per gram of soil was determined by counting the number of plaques cultivated from soil samples. Species were identified by morphology and the DNA sequences of 18S rRNA genes. Samples were obtained monthly from May 2012 to January 2013 inclusive.

In more detail, sampling occurred using two $100 \mathrm{~m}^{2}$ quadrats in Washidu $\left(35^{\circ} 3^{\prime} 33^{\prime \prime} \mathrm{N}, 138^{\circ} 53^{\prime} 46^{\prime \prime} \mathrm{E} ; 35^{\circ} 3^{\prime} 45^{\prime \prime} \mathrm{N}, 138^{\circ} 53^{\prime} 32^{\prime \prime} \mathrm{E}\right)$. Within each $100 \mathrm{~m}^{2}$ quadrat, nine sample points were established at $5 \mathrm{~m}$ intervals. From each sampling point, $25 \mathrm{~g}$ of soil was collected. Cellular slime molds were isolated from these samples as follows. First, one sample from each site was added to 25 $\mathrm{ml}$ of sterile water, resuspended, and then filtrated with sterile gauze. Next, $100 \mu \mathrm{l}$ of each sample solution was mixed with $100 \mu \mathrm{l}$ of HL5 culture medium containing Klebsiella aerogenes and spread onto KK2 agar. After two days of storage in an incubator at $22^{\circ} \mathrm{C}$, the number of plaques on each agar plate was enumerated and recorded. Note that the number of plaques corresponds to the total number of living cells at any possible stage of the life cycle. That is, the niche considered here is the set of propagable individuals of Dictyostelia; these are not arranged in any hierarchy or by stage in the life cycle. Also, note that we did not examine the age or size structure of organisms, since most of these were unicellular microbes. Mature fruiting bodies, consisting of cells from a single species, were collected along with information regarding the numbers of plaques in the regions in which each fruiting body was found. Finally, spores were used to inoculate either KK2 for purification or SM/5 for expansion. All analyses were performed within two weeks from the time of collection. The isolated species were identified based on 18S rRNA (SSU) sequences, which were amplified and sequenced using PCR/sequencing primers, as described in [Medlin et al., 1988] and the 
SILVA database (http://www.arb-silva.de/). The recipes for the media are described at http://dictybase.org/techniques/media/media.html.

\section{Results}

\subsection{Imaginary part of zeros for Riemann zeta function deciphering scaling hypothesis}

Drawing on the notion of critical phenomena of magnetic bodies, we developed a biological phase model [Adachi, 2019a]. To test the hypothesis, let us start from the empirical fact of critical indices in high isotropy for specific heat, spontaneous magnetization, and magnetic susceptibility $\alpha \approx-0.14 \approx-T_{1} / 100, \beta \approx 0.38 \approx T_{6} / 100$, and $\gamma \approx 1.375 \approx T_{47} / 100$, where $T_{n}$ represents imaginary values of Riemann $\zeta$ non-trivial zeros corresponding to $n$-th primes in ascending order. $\alpha+2 \beta+\gamma \approx 2$ is the empirical fact of the scaling hypothesis. If we apply this scaling hypothesis for magnetization to the PzDom model as an analogy,

$$
\ln P D^{N_{k}}=\ln P+(-1 / b)\left(-\Re(s) N_{k}\right)+i \Im(s) N_{k} / b,
$$

where $\alpha=\ln P$ for specific heat term, $\beta=-1 / b$ for the second term of spontaneous magnetization (being fractal; as shown later, 26 dimensions similar to a heterotic string theory or, Fake Monster Lie Algebra as supposed in [Borcherds, 1996, Kachru and Tripathy, 2017] in the sense of $\nabla$ (later indicated as related to $(p, v)$ in [Adachi, 2017]) and $\left.T^{7}\left(\times T^{2}\right)\right)$, which may become apparent when $p \equiv 1,3 \bmod 8$ and $p \equiv 1,3,4,9,10,12 \bmod 13$, considering $p=x^{2}+26 y^{2}$; from Castelnuovo's theorem, $X$ being a curve of even degree $d$ and genus $g$ in $\mathbb{P}^{3}$ not belonging to a plane, $g \leq \frac{1}{4} d^{2}-d+1=25$ when $d=12$ [Hartshorne, 1977], setting a dimensional limit to symmetries of a string theory), $\gamma=1 / b \Im(s)$ for magnetic susceptibility term. Energy $E=-\Re(s) N_{k}$, momentum $\mathbf{P}=\Im(s)^{2} N_{k}$, temperature $T=b$. The first two terms are obviously étale functions and the last term is a Frobenioid.

Furthermore, expected prime $l=\Re(s)$ values for species [Adachi, 2019b] exhibit right helicoid movement with constant $M_{z}+\hbar P_{z}$, where $M_{z}, P_{z}$ are $z$-components of angular momentum and momentum, and $l, \Im(s)=\bar{D}$ are radius of rotation and average linear rate of growth [Adachi, 2019b]. Taking $\hbar=1$, constant $l$, and a counterpart of mass as a constant measure for population/species density, $\bar{D}=N_{k} / E(N), \bar{D}+l \bar{D}$ is constant and $(1+l) \bar{D}=$ 
$C_{1} t+C_{0}$, where $t$ is a parameter and $C_{1}, C_{0}$ are constants. In this helicoidtype development of a ruled surface, $(l+1)$ can be defined as a counterpart of mass regarding $\bar{D}$ as a velocity. This development can explain a fractal structure [Adachi, 2019b] by adding a single dimension to the model. That is, with $(l, t)$ as auxiliary variables, we can take a minimal surface $(x, y, z) \in \mathbb{R}^{3}$ as $x=l \cos t, y=l \sin t, z=(1+l) \bar{D}=C_{1} t+C_{0}$ and the $z$-axis is the additional axis added as the fractal structure. This is exactly shown when $(l, t)$ generate a surface of minimal area as required for least actions. Note that considering von Neumann entropy $S_{v N}[\hat{\rho}]=-k \operatorname{Tr}[\hat{\rho} \ln \hat{\rho}], \hat{\rho}=\sum p_{i} \hat{P}_{\varphi_{i}}$, where $\varphi$ is a particular state and $\hat{P}$ is an orthogonal projection operator, maximizing von Neumann entropy means orthogonalization of the systems as producing orthogonal fractal dimensions. The nematic liquid crystal phase (as no fractal) or the cholesteric liquid crystal phase (as fractal of helicoid) is a candidate model for analogy.

Next, take $\sim 2$ as corresponding to the box dimension of $B, \operatorname{dim}_{B} B=2$, as the border of $\Re(s)$ distinguishing Minkowski measurable (structured by quantization) and Minkowski non-measurable (chaotic) spaces. $\alpha+2 \beta+\gamma \approx 2$ should be a fractal dimension and scaling parameters could be mapped to some sort of topological dimensions. Since $T_{1}, T_{6}, T_{47}$ correspond to $2,13,211$ of a particular category of the number of "unsplittable" interactions [Adachi, 2019a], $10^{2}=100$ and before interaction of the constituents, the root of this normalizing parameter should be $2 \times 5=10,(2+2 \times 13+211) / 10=23.9 \approx 24$. The importance of " 5 " is described below. Furthermore, summing up the merely self-interacting case with $p=1,240 / 10=24$. Since $\epsilon$-expansion of the renormalization group shows $(\alpha, \beta, \gamma)=(\epsilon / 6,1 / 2-\epsilon / 6,1+\epsilon / 6)$, a renormalization of a 12-dimensional system as in [Adachi, 2019b] indicates $\epsilon=12$ and $(\alpha, \beta, \gamma)=(2,-3 / 2,3)$, which corresponds to the dimensions of specific heat, spontaneous magnetization, and magnetic susceptibility. The sum of the dimensions of specific heat and magnetic susceptibility is 5 , and duplication for the dimension of spontaneous magnetization is -3 . The latter is diminished dimensions that contribute to a fractal.

If we constitute a Fuchs-type differential equation from the system, a necessary condition for the sum of characteristic exponents $\lambda$ is $\sum \lambda=240$. This is equal to $\sum_{a \in S} \sum_{k=1}^{n} \lambda_{a, k}=\frac{n(n-1)(\# S-2)}{2}$ when \#S is the number of elements in $S$. Splitting 240 into $\{1,2,13,13,211\}$ means $n=5$ and $\# S=26$ of the heterotic string theory. This necessary condition is sufficient for that the G.C.D. of $\{1,2,13,13,211\}$ is 1 and the Fuchs-type differential equation, 
i.e., a hypergeometric differential equation [Adachi, 2019a], does really exist. The dimension for the equation derived in Srinivasa Ramanujan (1916?) in an unpublished manuscript is as follows:

$$
F(z)=q \prod_{n=1}^{\infty}\left(1-q^{n}\right)^{2}\left(1-q^{11 n}\right)^{2}=\sum_{n=1}^{\infty} c(n) q^{n}
$$

Consider a stress tensor $T^{i k}$. Therein, 13 independent elements could be regarded as a cyclic group of a power of $q$, as in

$$
\left\{W, S_{x / c}, S_{y / c}, S_{z / c}, \sigma_{x x}, \sigma_{x y}, \sigma_{x z}, \sigma_{y x}, \sigma_{y y}, \sigma_{y z}, \sigma_{z x}, \sigma_{z y}, \sigma_{z z}\right\} .
$$

This is on the basis of 13 dimensions in analogy to spontaneous magnetization. Without $W$, it becomes 12 dimensions (as Teichmüller space related to torus, $6 g-6=12, g=3$ for two self-interacting terms and one heterointeracting term) and their interaction leads to 24 dimensions or weights (as Teichmüller space related to torus, $6 g-6=24, g=5$ for $\Re(s)=5$ ). Analogous to electric field and magnetic field, $\mathbf{E} \perp \mathbf{H}, E=H$, the tensor cannot be diagonalized and there is no étale element. Note that the weight 24 appears at free energy of canonical distribution in quantum theory as follows:

$$
F=F_{c l}+\frac{1}{24 T^{2}} \sum_{i} \frac{1}{m_{i}}\left\langle\left(\frac{\partial U}{\partial q_{i}}\right)^{2}\right\rangle .
$$

$F \rightarrow T_{e f f}, F_{c l} \rightarrow T, 24 \rightarrow 12$ transitions are also derived as expected. Furthermore, $\alpha=\left(2 a_{s}-1\right) / a_{s}, \beta=\left(1-b_{s}\right) / a_{s}, \gamma=\left(2 b_{s}-1\right) / a_{s}, a_{s}=1 / 2$, and $b_{s}=3 / 4$ for the classical case. Let us consider an $X^{2}+X Y+Y X+Y^{2}$ system with a single dimension equipartitioned to each, $1 / 4 . \quad a_{s}=1 / 2$ means the hetero interaction terms $X Y, Y X$ should diminish to zero. This is as per the PzDom model [Adachi, 2019a], the harmonic neutrality with $\Re(s)=1$. However, once the system moves forward to the cooperative part of $1<\Re(s)<2$, it first develops commensalism, and $X Y$ should be validated as $1 / 4$ but $Y X$ is still zero, with $b_{s}=3 / 4$. Thus, the development of commensalism would result in the development of cooperation with the parameter equal to 1 , moving into $\Re(s)>2$ with no further interaction with phase transition in an analogy to an ideal gas. For example, the $P_{W}=\frac{2}{3} u$ relation ( $P_{W}$ is a pressure and $u$ is a density of internal energy) shows a $2 / 3$ norm for geodesics of a Selberg zeta function in non-interacting mode of $\Re(s)=2,3$ [Adachi, 2019a]. Note that 
a general law between pressure and energy, $P_{W}<\frac{E}{3 V}$, implies a norm of $1 / 3$ derived from the trichotomy of the $X^{2}+X Y+Y^{2}$ system [Adachi, 2019a]. The norm $|N(p)|=1 / 3$ appears in each density of population/species. For further development stricter than an ideal gas, consider an analogy to van der Waals law:

$$
P_{W}=\frac{N T_{W}}{V-N b_{W}}-\frac{N^{2} a_{W}}{V^{2}}
$$

which results in critical points $T_{c r}=\left(\frac{2}{3}\right)^{3} \frac{a_{W}}{b_{W}}, V_{c r}=3 N b, P_{c r}=\left(\frac{1}{3}\right)^{3} \frac{a_{W}}{b_{W}^{2}}$ (here $N$ is reduced number of molecules). $\frac{1}{3} X^{2}+\frac{0}{3} X Y+\frac{1}{3} Y^{2}$ model [Adachi, 2019a] is applicable for this case and the critical phenomena are deeply involved in situations analogical with gas. Empirically, $q / T_{c r} \sim 10$ when $q$ is the latent heat for vaporization and temperature is significantly lower than the critical temperature, indicating $T_{c r}$ is the border between the $\frac{0}{3} X Y$ world and the $\frac{1}{3} X Y$ world. $5+5=10$ was observed for development to $\Re(s) \geq$ 5 (empirically observed in October in the Washidu East quadrat between Polysphondylium pallidum and Dictyostelium purpureum[Adachi, 2019a]; this value is exactly the critical temperature; $\left(a_{W}, b_{W}\right) \sim(2700,0.53),(82000,16)$ for each; the latter is 30 times the former, and $P$. pallidum may act as $l=2$ part and $D$. purpureum may act as $l=3$ part ( 2 main fractal dimensions with 1 vibrational dimension, later indicated); both of the species are shrinking because $\left.P_{W}<0\right)$.

However, consider an additional critical index for magnetic charge, $\delta(=$ $3)$. This is reasonable because population/species density should be 3 -dimensional, and a single dimension would be equipartitioned to the 3 dimensions for the counterpart of the magnetic charge. $\alpha+2 \beta+\gamma+\delta \geq 5$ for the classic case (Rushbrooke inequality), and a prime equal to or larger than 5 would lead to the deeper mathematical logic behind Inter-Universal Teichmüller Theory [Mochizuki, 2019a, Mochizuki, 2019b, Mochizuki, 2019c, Mochizuki, 2019d]. This reflects a hierarchical structure induced by $\mathbb{R}^{3} \times \mathbb{R}^{3}=\mathbb{R}^{6}$ directly produced by another $\mathbb{R}^{3}$ structure that already belongs to $\Re(s) \geq 5$, resulting in 9 dimensions. This becomes prominent in $\Re(s) \geq 5$. That is, for development of a system that goes beyond $\operatorname{dim}_{B} B=2$, a different interaction partner for development beyond $\Re(s)=5(2 \times 5=10)$ is needed. Or, considering the $p=x^{2}+5 y^{2}$ case leadings to $p \equiv 1,9 \bmod 20$ would potentially be sufficient, which is empirically not observed in [Adachi, 2019a]. This leads to a Navier-Stokes-type interaction equation with "viscosity" as entanglement of different subgroups and the dimension 24 is thus linked to critical phenomena 
Table 1: $\phi^{\prime}$ values.

\begin{tabular}{|l|l|l|l|l|}
\hline$\phi^{\prime}$ & $P$. pallidum 1 & $P$. violaceum 1 & P. pallidum 2 & P. violaceum 2 \\
\hline WE June & $1.00 \mathrm{E}+168$ & $2.3 \mathrm{E}+272$ & $-2.1 \mathrm{E}+167$ & $-2 \mathrm{E}+271$ \\
\hline WE November & $4.7 \mathrm{E}+69$ & $7 \mathrm{E}+186$ to $4 \mathrm{E}+188$ & $-1.06 \mathrm{E}+69$ & $-1 \mathrm{E}+185$ to $-5 \mathrm{E}+186$ \\
\hline
\end{tabular}

WE: Washidu East quadrat; P. pallidum: Polysphondylium pallidum; P. violaceum: Polysphondylium violaceum. 1,2 denote $\varepsilon=-\frac{5}{24} \omega_{0}, \frac{1}{24} \omega_{0}$ cases, respectively.

[Adachi, 2019a]. Considering Mathieu's equation

$$
\ddot{x}+\omega_{0}^{2}\left[1+\cos \left(2 \omega_{0}+\varepsilon\right) t\right] x=0,
$$

the resonance in the neighborhood of outer vibration $\gamma_{M}=\omega_{0}$, the limits of instable regions for parameter resonance are $\varepsilon_{M}=-\frac{5}{24} \omega_{0}, \frac{1}{24} \omega_{0}$. These calculations clearly suggest an interpretation of critical phenomena by a $24-$ dimensional system and an accompanying resonance by an $\Re(s)=5$ system. For the Mathieu (Hill) equation, a general solution would be $x(z)=$ $e^{\mu_{M} z} x_{1}(z)+e^{-\mu_{M} z} x_{2}(z)$ when $x_{1}, x_{2}$ would bear periodic functions such as $\omega_{0}^{2}\left[1+\cos \left(2 \omega_{0}+\varepsilon_{M}\right) t\right]$. At least locally, this equation means transfer of étale from one to the other. Note that the étale is calculable with

$$
\cosh \mu_{M} T_{M}=\phi_{2}\left(\frac{T_{M}}{2}\right) \phi_{1}^{\prime}\left(\frac{T_{M}}{2}\right)+\phi_{1}\left(\frac{T_{M}}{2}\right) \phi_{2}^{\prime}\left(\frac{T_{M}}{2}\right),
$$

where $\phi$ are particular solutions for $x$ and $T_{M}=2 \pi /\left(2 \omega_{0}+\varepsilon_{M}\right)$. Calculations for $\phi^{\prime}$ values indicate that $\varepsilon_{M}=-\frac{5}{24} \omega_{0}$ is the dominant mode which empirically prevails in Dictyostelia (Table 1).

In analogy to rigid bodies (uniform sphere of radius $a$ ) as Monsters, $I=\frac{2}{5} \mu a^{2}$ is the moment of inertia and $I \frac{d \Omega_{z}}{d t}=K_{z}$ is the $z$-component of the moment of force in the fractal axis. This equation shows a Monster of $l=2$ guided by $\Re(s)=5$ resonance. Two Monsters with boundaries and a single dimension for additional fluctuation (the normal vibration for two atoms), $2+2+1=5$ means successful construction of an $\Re(s)=5$ system. If the energy for the vibration is strong, a Hodge-Kodaira decomposition type observation and time development model [Adachi, 2019a] shows the boundary of interaction as a hyperboloid of one sheet, a connected relation. On the other hand, weak vibration shows that as a hyperboloid of two sheets, split (of course when the sheet reaches the origin of coordinates, it is a cone, indicating observation). Additionally, the anisotropic 
situation of critical phenomena could be modified by the difference between an almost analogical situation to Helmholtz free energy without migration (PzDom) and Gibbs free energy with migration, resulting in slightly lower values of indices compared with isotropic critical phenomena. In analogy to fluid mechanics, a long shallow-water wave would be observed in a neighbor of $\partial B$, while a short deep-water wave would be observed close to the origin of coordinates. $\lambda_{m}=2 \pi / N(T)$ [Adachi, 2019a] would be a particular wave and $\lambda<\lambda_{m}$ would be a capillary wave with the effects from surface $(\partial A)$ tension $(A \subset B)$ being prominent. On the other hand, if $\lambda>\lambda_{m}$, the gravity wave with effects from $D$ is prominent. That is, $D$ affects regions close to the boundary and inner structures have their own substructures depending on the borders. For instance, vorticity can be analyzed by persistent homology[Kramár, 2016, Kashiwara and Schapira, 2018], that is, $p$ [Adachi, 2019a]. Note that $\Re(s)$ is an étale cohomology [Adachi, 2019b] and dual to $\Im(s)$. Homology is related to inner structure, while cohomology is related to the border. For example, consider the Poisson-Schwarz integral formula

$$
f\left(z_{0}\right)=i C_{I}+\frac{1}{2 \pi i} \int_{\partial A} \Re[f(z)] d K, f\left(z_{0}\right)=C_{R}+\frac{1}{2 \pi} \int_{\partial A} \Im[f(z)] d K,
$$

where $H$ is a complex velocity potential with $\left(z_{0}, a \in A\right)$ as source, sink of strength $1, G$ is a complex velocity potential with $z_{0}$ exhibiting a vortex filament of strength $1, K=H\left(z ; z_{0}, a\right)+i G\left(z, z_{0}\right), C_{R}=\Re[f(a)]$, and $C_{I}=$ $\Im[f(a)]$. These formulae are useful for analyzing inner structures or boundary behavior, to the extent that the situation is regular and singularities can be neglected.

\subsection{A master automorphic form}

Automorphic form is an invariant meromorphic function for linear transformation groups. For a modular group, it is called a modular function. A modular function

$$
\lambda(\tau)=\lambda\left(\frac{a \tau+b}{c \tau+d}\right),\left(\begin{array}{ll}
a & b \\
c & d
\end{array}\right) \equiv\left(\begin{array}{ll}
1 & 0 \\
0 & 1
\end{array}\right)(\bmod 2)
$$

is a permutation from $(0,1, \infty) \rightarrow(1, \infty, 0)$, hence $\lambda^{3}=i . d$. Thus an observant, an observer, and a limit constitutes a system of interest in 3- 
dimensional space. For the modular group,

$$
J(\tau)=\frac{4}{27} \frac{\left(1-\lambda+\lambda^{2}\right)^{3}}{\lambda^{2}(1-\lambda)^{2}}=\frac{-4\left(e_{1} e_{2}+e_{2} e_{3}+e_{3} e_{1}\right)^{3}}{\left(e_{1}-e_{2}\right)^{2}\left(e_{2}-e_{3}\right)^{2}\left(e_{3}-e_{1}\right)^{2}}
$$

is the automorphic form and a specific $\Delta$ of $\Delta \oplus \Delta^{\prime}=[-1,1] \subset \mathbb{H}$ would be mapped to $\mathbb{H}$. In future, $0 \rightarrow 1,1 \rightarrow \infty$, and $\infty \rightarrow 0$ would be achieved [Ahlfors, 1979]. Note the case that a trace of $\lambda$ is 2 , which results in the existence of a master form of

$$
\rho_{G}\left(c_{G}\right)=c_{G} \mathrm{Id}_{W}
$$

when $\operatorname{Id}_{W}=|J(\tau)|$ is an identity mapping (Stone-von Neumann theorem; [Stone, 1930, von Neumann, 1931, von Neumann, 1932, Stone, 1932]). This is the $\varnothing=\partial B$ case. $J(\tau)=1$ can be recursively defined by the relations above with any particular time point as the initial value. Assuming a primitive recursive function would not require such an initial value over infinity, if one has all the information incorporated to $J(\tau)$. However, in the PzDom model [Adachi, 2019a], the final output of the model in static state would be $N_{k}=a, b=0, D=1, H(t)=1 / 2, \Re(s)=0$, and $\Im(s)=1$ (note that $N_{k}=a-b \ln k$ case, different from the parameters $a, b$ in the previous matrix) and thus $s=i$. For constant $s$ multiplication as a shift map for time development, the resultant $\lambda_{s}$ would be

$$
\left(\begin{array}{ll}
i & 0 \\
0 & 1
\end{array}\right)(\bmod 2)
$$

For $\operatorname{Id}_{W}=J(\tau), \tau=\frac{i \pm \sqrt{7}}{2}$ and this goes beyond the border of $|\tau|=2$ as expected, to cross the border of $\Re(s)=2$ in the PzDom model [Adachi, 2019a]. Therefore $\varnothing \neq \partial B$ (open model) beyond the border can be introduced by this $\tau=\frac{i \pm \sqrt{7}}{2}$ acting as a seed of $\partial B$ (closed model). The virial theorem shows a relation between kinetic energy $T_{v}$ and potential energy $U$ as $\bar{U}=2 E /(k+2)$ and $\bar{T}_{v}=k E /(k+2)(E$ is total energy, and barred symbols are averages with $k$-th order of a constant for similarity) with homogeneous potentials and coordinates in finite regions. A system of a counterpart of force with the inverse squared law shows an elliptic orbit with $t^{\prime} / t=\left(l_{o}^{\prime} / l_{o}\right)^{3 / 2}$, where $l_{o}$ is a locus of the orbit. $k=3 / 2, \bar{U}=\frac{4}{7} E, \bar{T}_{v}=\frac{3}{7} E$. Masses are related by their square roots and $\sqrt{\bar{U}}=\frac{2}{\sqrt{7}} \sqrt{E}=\sqrt{E} / l$ for $\Re(\tau)=\frac{\sqrt{7}}{2}$. When $\sqrt{E} \in \mathbb{Z}, \sqrt{\bar{U}}$ is homomorphic to $\mathbb{Z} / l \mathbb{Z}$ and thus $\sqrt{\bar{T}}$ is homomorphic to $l \mathbb{Z}$, as in the Chinese Remainder Theorem. Similar logic holds for a squared potential, $D^{2}$ space with 
$\Re(s)=2$. Thus, $(D, s) \cong(\sqrt{E}, \tau)$, and there is a $2 \leftrightarrow \frac{\sqrt{7}}{2}$ correspondence. It is notable that for elliptic integrals of the first kind, $K=\int_{0}^{1} \frac{d z}{\sqrt{\left(1-z^{2}\right)\left(1-k^{2} z^{2}\right)}}$ (Legendre canonical form), moduli $\left(k, k^{\prime}=\sqrt{1-k^{2}}\right)=(i, \sqrt{2})$ are a pair of the periods of the inverse functions $z$ of canonical forms $K=\frac{\sqrt{2} \Gamma^{2}(1 / 4)}{8 \sqrt{\pi}}$ and $\left(4 K, 2 i K^{\prime}\right)=(5.244,2.622+2.622 i)$. Branch points for $\sqrt{1-z^{2}}$ are $(1.311,3.933)$. Since the sum of all the residues in a periodical parallelogram of elliptic functions is zero, if something non-zero is observed, a periodical parallelogram becomes unbounded and time is infinite. Thus, rotation by $i$ and $|\tau|=\sqrt{2}$ are a pair, considering interacting elliptic functions $\left(i^{2}=-1, \sqrt{2}^{2}=2\right)$ for them. Weierstraß $\wp=\left(\frac{K}{\omega_{1}}\right)^{2}\left(\frac{1}{z^{2}}-\frac{1+k^{2}}{3}\right)$ means $k=i$ is a Frobenioid (monoid and morphism as an action induced by the ring homomorphism) only with the $\frac{1}{z^{2}}$ term, and the remaining -1 with $k=\sqrt{2}$ is an étale (Fig. 1). For $\tau=i$, it is notable that $R=\mathbb{Z}[i]$ and $R^{*} \cong \mathbb{Z} / 4$. The group of automorphisms of a set $X$ has order 4 , and $j=1728$ (char $k \neq 3$ ). Thus, $J(\tau)=1 . \Lambda=\mathbb{Z} \oplus \mathbb{Z} i$ and $g_{3}=-g_{3}=0$; thus, $X$ is $y^{2}=x^{3}-A x$ [Hartshorne, 1977], rendering $-g_{3}$ as étale. This is equivalent to the Berger theorem [Kedlaya, 2010], where the functor $D_{\text {rig }}^{\dagger}$ from the category of continuous representations of $G_{K}$ on finite-dimensional $\mathbb{Q}_{p}$-vector spaces to the category of étale $(\phi, \Gamma)$-modules over $\mathbf{B}_{\mathrm{rig}, K}^{\dagger}$ is an equivalence of categories, when the situation is as supposed in rigid geometry [Adachi, 2017]. That is, there exists such a continuous representation $G_{K}$ considering the torus above. An example of a (Lie) group that corresponds to $G_{K}$ is as follows. Let $v_{K}: \Gamma_{K} \rightarrow v \rightarrow \mathbb{Z}_{p}^{\times}$denote the cyclotomic character; that is, for all negative integers $m$ and all $N_{k}=\gamma_{\nabla} \in \Gamma_{K}, \gamma_{\nabla}\left(\zeta_{p^{m}}\right)=\zeta_{p^{m}}^{v_{K}}$. In this case, we can compute

$$
\nabla=\frac{\ln \gamma_{\nabla}}{\ln v}
$$

as an endomorphism of $D_{\text {rig }}^{\dagger}$, using the power series for logarithms. We can regard $\Gamma_{K}$ as a one-dimensional $p$-adic Lie group over $\mathbb{Z}_{p}$ because of the continuous nature of $v$ and then $\nabla$ is an action of the Lie group. In this way, [Adachi, 2019a] defines a discrete group $N_{k} / l \mathbb{Z}$ and here we can define a continuous Lie group $\nabla$ over $\mathbb{Z}_{p}=l \bmod p$ as a $p$-adic Hodge theory that relates étale cohomology to de Rham cohomology [Mochizuki, 1999]. We can also draw a short exact sequence,

$$
(\sim 0) \rightarrow p \rightarrow v \rightarrow \nabla \rightarrow(\sim 0) .
$$


Frobenioids and étales are thus classified as continuous and discrete natures of corresponding groups. As later indicated, this is why renormalization neglecting fractal structures is sufficient for interpreting theories based on Lie groups in physics. However, for integrative models, we require discrete fractal structures from étale properties. Thus, $(p, l)$ or $(p, v)$ could be regarded as two dimensions of a Young tableau and the numbers constitute an order of appearances in the system. It is notable that multiplications for $p, l, v$ result in additions by log structures.

\subsection{Communication among different sets (of Monsters) described by an integrated model with a master Lagrangian}

Consider a finite covering of $B$ [Adachi, 2019b] as a compact space such as a compact Riemann surface (or any infinite series of a point which has an accumulation point with identity; Bolzano-Weierstraß theorem; Artin perspective). Therein, the order limit of finite simple groups is at most that of a Monster group (maximizing entropic information while minimizing free energy, Noetherian perspective); i.e., if the order goes beyond the value, it becomes unavoidably not simple and the system will split. This causes $\varnothing=\partial B$ in that situation. For example, take an absolute zeta function $p^{\prime}=s / w$ [Adachi, 2019a]. It belongs to a distribution space of $\left(\mathscr{D}_{L^{p^{\prime}}}\right)$ and it should be a finite sum of differential coefficients of functions $\in L^{s}$ [Schwartz, 1966]. However, if there is still an infinitely ordered Sato hyperfunction beyond the Schwartz distributions and boundaries [Sato, 1959, Sato, 1960], communications among different Monsters (analogical to rigid bodies; angular velocity $\Im(s)$ is independent of the coordinate system while translational motion or a fractal dimension $\Re(s)$ is dependent on a particular coordinate system such as an observer [Adachi, 2019a]; i.e., the nonholonomic world of relativity versus the holonomic world of quantum mechanics) are still possible. These communications render it plausible to bind up the different Monsters to a certain system, in discrete terms, not in the sense of manifolds. Let us consider the match of Steiner circles, or for elliptic integrals considered later as $D$, Poncelet's poristic polygons including Steiner's series [Emch, 1899-1900, Emch, 1900-1901] in two different Monsters. For the most effective communications, a non-Euclid distance, such as a Lorentz metric, should be selected, multiplied by $\sqrt{-1}$ to accommodate hyperfunctions. 


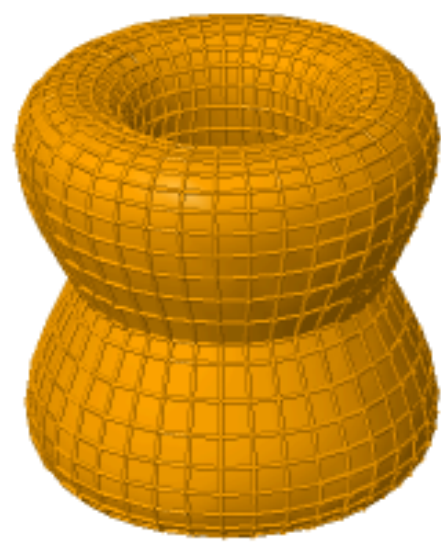

Figure 1: The torus of moduli $(i, \sqrt{2})$. 
The best communication should entail sending out the information of $J(\tau)$ to match the series. Ideally this is between $\Delta\left(\cong B_{1}\right)$ and $\Delta\left(\cong B_{2}\right)$ via $\tau=\frac{i \pm \sqrt{7}}{2}$. Here, the Routh function is apparently useful for communication from a particular Monster with a Lagrangian (least action for calculating the trajectory):

$$
R\left(q, p_{m}, \xi, \dot{\xi}\right)=p_{m} \dot{q}-L .
$$

As later shown, given a momentum and $q, \mathbf{P}=\Im(s) \phi, q=D$, and the relative entropy $S=\ln \Delta \Gamma=\ln \frac{\Delta \mathbf{P} \Delta q}{(2 \pi \hbar)^{3}}$ and setting $(2 \pi \hbar)^{3}$ as a volume of line integral of action, $k$ becomes uncertainty per volume. The least action is thus connected to maximum entropy. In other words, a least action maximizes a Minkowski metric $s_{M}$ [Adachi, 2019b]) accompanied by $\xi$ to another Monster with a Hamiltonian (energy for a status) accompanied by $q$; e.g., a Carnot cycle of a particular Monster $\left(M_{C}\right)$ gives work to another Monster $\left(M_{W}\right)$, the latter sucking up free energy from the former. Dealing with a Frobenioid related to $q$ is straightforward from the viewpoint of Kobayashi (for the étale function, refer to Inter-Universal Teichmüller Theory [Mochizuki, 2019a, Mochizuki, 2019b, Mochizuki, 2019c, Mochizuki, 2019d]). Simply, we took the Dirac picture and $H=H_{0}+H^{\prime}$, where $H, H_{0}$, and $H^{\prime}$ are Hamiltonian, partly independent, and partly dependent of time. $M_{W}, M_{C}$ correspond to Hamiltonians of $H_{0}, H^{\prime}$. This interaction picture (abbreviated to $I$; the Schrödinger picture is abbreviated to $S$ ) leads to

$$
\left|\alpha_{I}(t)\right\rangle \equiv e^{i H_{0 S} t}\left|\alpha_{S}(t)\right\rangle, \beta_{I}(t) \equiv e^{i H_{0 S} t} \beta_{S} e^{-i H_{0 S} t}, i \frac{d}{d t}\left|\alpha_{I}(t)\right\rangle=H_{I}^{\prime}\left|\alpha_{I}(t)\right\rangle,
$$

where $\beta=1 / b$ is inverse temperature. For example, if we set $\alpha^{\prime \prime}(\omega) d \omega=$ $\alpha^{\prime \prime}(b)\left|\alpha_{I}(b)\right\rangle=\Im(s) D$ and $\omega \alpha^{\prime \prime}(\omega)=\left\langle\alpha_{I}(b)\right| \alpha^{\prime \prime}(b)=\Im(s) D, \int_{0}^{\infty} \omega \alpha^{\prime \prime}(\omega) d \omega=$ $\frac{i \pi}{2}\langle\{\dot{\hat{D}} \hat{D}\}\rangle=0$ leads to commutative relation in the Poisson bracket $(=m$ of quantization number in [Adachi, 2019a]). $\pi m / 2$ could be a retarded Green function, or from an elliptic function perspective, Legendre's relation $\eta_{1} \omega_{3}-\eta_{3} \omega_{1}=\pi m / 2$. In this case, when the time of $\hat{D}$ is earlier (later) than the time of $\dot{\hat{D}}$, it is non-zero (zero). Generally this is the case with Dictyostelia, excepting Polysphondylium violaceum in November at the Washidu East quadrat [Adachi, 2019a]. This operation predicts the presence of an irreversible process when the formula is non-zero. If we fix $\hat{D}$, $\dot{\hat{D}}$ starts from the Green function being zero and after it crosses the time 
for $\hat{D}$, the Green function becomes apparent. Note that in this case, we set $\varepsilon=\mu=D / \Im(s)\left(=\alpha^{\prime \prime} / \Im(s)^{2}\right)$. The distribution of $\varepsilon, \mu$ in different ratios depending on $\varepsilon \mu=(D / \Im(s))^{2}$ results in a distribution of étale and Frobenioid analogous to the electric and magnetic forces, respectively. When $t \rightarrow \infty$, $H(t)=1 / 2$ and since $m \in \mathbb{Z}, \hat{D}^{2} \in 2 \mathbb{Z}$, fulfilling the condition for crossing the border $\Re(s)=2$. For further development, some forces analogical to that of physics as inverse square laws, such as gravity and electric fields, can be defined on $D$-space. $M=\phi / \sqrt{1-(H(t) D)^{2} / \Im(s)^{2}}$ is analogical to mass, or electric or magnetic charge. The reason for introducing squares will be discussed later. For the electric force,

$$
\mathbf{F}_{e}=\frac{\operatorname{sign}(M)}{4 \pi \varepsilon} \frac{M_{i} M_{j}}{D^{2}} \mathbf{e}_{\mathbf{F}}
$$

could be defined. Note that the $\operatorname{sign}(M)$ is set at plus when the force acts to increase the number of individuals of particular $M_{i}$, and set at minus when the force acts to decrease the number of individuals of particular $M_{i}$. $i=j$ means self-interaction. Note that $\varepsilon=D / \Im(s)$ in this case. This holds similarly for the magnetic force, with $\mu=D / \Im(s)$. For the gravity force,

$$
\mathbf{F}_{g}=\frac{\operatorname{sign}(M)}{4 \pi k_{g}} \frac{M_{i} M_{j}}{D^{2}} \mathbf{e}_{\mathbf{F}}
$$

could be defined. Note again that the $\operatorname{sign}(M)$ is set at plus when the force acts to increase the number of individuals of particular $M_{i}$, and set at minus when the force acts to decrease the number of individuals of particular $M_{i}$. $i=j$ means self-interaction. Note that $k_{g}=D / \Im(s)$ in this case. Although this is apparently recognizable as a classical Newton equation, note that $k_{g}$ is a function of $D$, not a constant. The gravity, electric, or magnetic force is $\mathbb{R}^{3} \rightarrow \mathbb{R}^{2}$ projection of a more generalized form:

$$
\mathbf{F}_{s}=\frac{\operatorname{sign}(M) \Im(s)}{4 \pi} \frac{M_{i} M_{j}}{D^{3}} \mathbf{e}_{\mathbf{F}}
$$

and fixing a single dimension of $D$ as $k_{g} \Im(s)$ or $\varepsilon \Im(s)$ results in the gravity or electric force. Similar logic also applies for the magnetic force.

Now consider a Schwarzschild solution analogy to a black hole. The entropy of the solution would be $S_{B H}=c^{3} A / 4 G=\pi D 4 \pi(\Im(s) D)^{2}$, which represents a $\pi D$ increase of a sphere with radius $\Im(s) D$. The Planck-length is $\ell_{P}=1 / \sqrt{4 \pi D}$. The information of this solution is roughly accumulated in 
Table 2: $\lambda_{J}$ values compared to $D$ (Washidu East).

\begin{tabular}{|l|l|l|l|l|l|l|l|}
\hline$\lambda_{J}$ & P. pallidum & D. purpureum & P. violaceum & D & P. pallidum & D. purpureum & P. violaceum \\
\hline May & & & & May & & & \\
\hline June & 2.358 & 0.4255 & 1.852 & June & 1.005 & 1.013 & 1.009 \\
\hline July & & & & July & & & \\
\hline August & & & & August & & & \\
\hline September & 1.472 & 5.324 & & September & 1.003 & 1.003 & \\
\hline October & 1.100 & 6.623 & & October & 1.003 & 1.003 & \\
\hline November & 2.253 & & 0.2943 & November & 1.013 & & 1.036 \\
\hline December & & & & December & & & \\
\hline January & & & & January & & & \\
\hline
\end{tabular}

P. pallidum: Polysphondylium pallidum; D. purpureum: Dictyostelium purpureum; P. violaceum: Polysphondylium violaceum. Numbers in red indicate perturbations larger than $\lambda_{J}$. Blank cells signify not calculable.

this minimal surface. The Schwarzschild radius is $r_{H}=2 G M=\Im(s) M / 2 \pi D$ and it is a quotient of a momentum $\Im(s) M$ to a circumference $2 \pi D$. The Hawking temperature is $T_{H}=\frac{1}{8 \pi G M}=\frac{\pi D}{2 \Im(s) M}$. Thus, species $M$ is accompanied by this trapping radius and a temperature analogical to the black hole. Jeans wave number $k_{J}$, wave length $\lambda_{J}$, and mass $M_{J}$ would be

$$
k_{J}=\sqrt{\frac{N_{k} \Im(s)}{b D}}, \lambda_{J}=2 \pi \sqrt{\frac{b D}{N_{k} \Im(s)}}, M_{J}=\frac{4}{3} \pi^{4} \sqrt{\frac{1}{N_{k}}\left(\frac{b D}{\Im(s)}\right)^{3}} .
$$

Perturbations larger than $\lambda_{J}, M_{J}$ would admit developments of species dynamics (Tables $2-5$ ). This analogy to celestial objects is developed into an H-R plot of species (Fig. 2). There are three distinct phases: $b$ values under $\sim 300$ denoting a chaotic situation, $b$ values above $\sim 700$ with an upper distribution $(N>600)$ for an adapted situation, and $b$ values above $\sim 700$ with a lower distribution $(N<200)$ denoting a disadapted situation. This type of plot is useful to discriminate the phase of the species.

For further investigation, consider an analogue of radius of action in the nuclear force, $\lambda \approx \frac{\hbar}{M c}=\frac{1}{M \Im(s)}$ and as $D \sim 1$ we can set $\lambda r_{H}=1$. Thus when $D>r_{H}$, there is an interaction between the Monsters analogous to a Schwarzschild black hole, which is likely to be involved in Yukawa-type potential. When $D \leq r_{H}$, it is not possible to get outside the Monster. If $r_{H} \ll 1$, there is an attraction force to develop the fractal dimension.

Furthermore, considering an inverse cube of $\frac{1}{3} \Im(s) D$ results in a case similar to quantum color dynamics (QCD) (equals the average of the square of 
Table 3: $\lambda_{J}$ values compared to $D$ (Washidu West).

\begin{tabular}{|l|l|l|l|l|l|l|l|}
\hline$\lambda_{J}$ & $P$. pallidum & D. purpureum & P. violaceum & D & P. pallidum & D. purpureum & P. violaceum \\
\hline May & & & & May & & & \\
\hline June & & & & June & & & \\
\hline July & 1.634 & 2.703 & 0.3881 & July & 1.006 & 1.003 & 1.008 \\
\hline August & 1.476 & 5.127 & & August & 1.002 & 1.002 & \\
\hline September & 0.7980 & 1.752 & 4.556 & September & 1.003 & 1.003 & 1.000 \\
\hline October & 1.478 & & 5.158 & October & 1.003 & & 1.003 \\
\hline November & & & & November & & & \\
\hline December & & & & December & & & \\
\hline January & & & January & & & \\
\hline
\end{tabular}

P. pallidum: Polysphondylium pallidum; D. purpureum: Dictyostelium purpureum; P. violaceum: Polysphondylium violaceum. Numbers in red indicate perturbations larger than $\lambda_{J}$. Blank cells signify not calculable.

Table 4: $M_{J}$ values compared to $N$ (Washidu East).

\begin{tabular}{|l|l|l|l|l|l|l|l|}
\hline$M_{J}$ & P. pallidum & D. purpureum & P. violaceum & $N$ & P. pallidum & D. purpureum & P. violaceum \\
\hline May & & & & May & & 75.56 & \\
\hline June & 841.7 & 8.423 & 174.5 & June & 122.6 & 208.9 & 52.44 \\
\hline July & & & & July & 1282 & & \\
\hline August & & & & August & 1561 & & \\
\hline September & 1504 & 8429 & & September & 900.6 & 106.7 & \\
\hline October & 745.3 & 5289 & & October & 1069 & 34.78 & \\
\hline November & 359.1 & & 1.345 & November & 60.00 & & 100.8 \\
\hline December & & & & December & 189.6 & & \\
\hline January & & & & January & 28.90 & & \\
\hline
\end{tabular}

P. pallidum: Polysphondylium pallidum; D. purpureum: Dictyostelium purpureum; P. violaceum: Polysphondylium violaceum. Numbers in red indicate perturbations larger than $M_{J}$. Blank cells signify not calculable. 
Table 5: $M_{J}$ values compared to $N$ (Washidu West).

\begin{tabular}{|l|l|l|l|l|l|l|l|}
\hline$M_{J}$ & $P$. pallidum & D. purpureum & $P$. violaceum & $N$ & P. pallidum & D. purpureum & P. violaceum \\
\hline May & & & & May & & 82.67 & \\
\hline June & & & & June & 146.7 & & \\
\hline July & 182.6 & 2220 & 9.795 & July & 80.00 & 214.8 & 320.0 \\
\hline August & 2241 & 12760 & & August & 1330 & 180.78 & \\
\hline September & 215.3 & 216.8 & 32130 & September & 809.2 & 77.00 & 648.9 \\
\hline October & 1350 & & 7666 & October & 798.8 & & 106.7 \\
\hline November & & & & November & 335.6 & & \\
\hline December & & & & December & 711.1 & & \\
\hline January & & & January & 99.00 & & \\
\hline
\end{tabular}

P. pallidum: Polysphondylium pallidum; D. purpureum: Dictyostelium purpureum; P. violaceum: Polysphondylium violaceum. Numbers in red indicate perturbations larger than $M_{J}$. Blank cells signify not calculable.

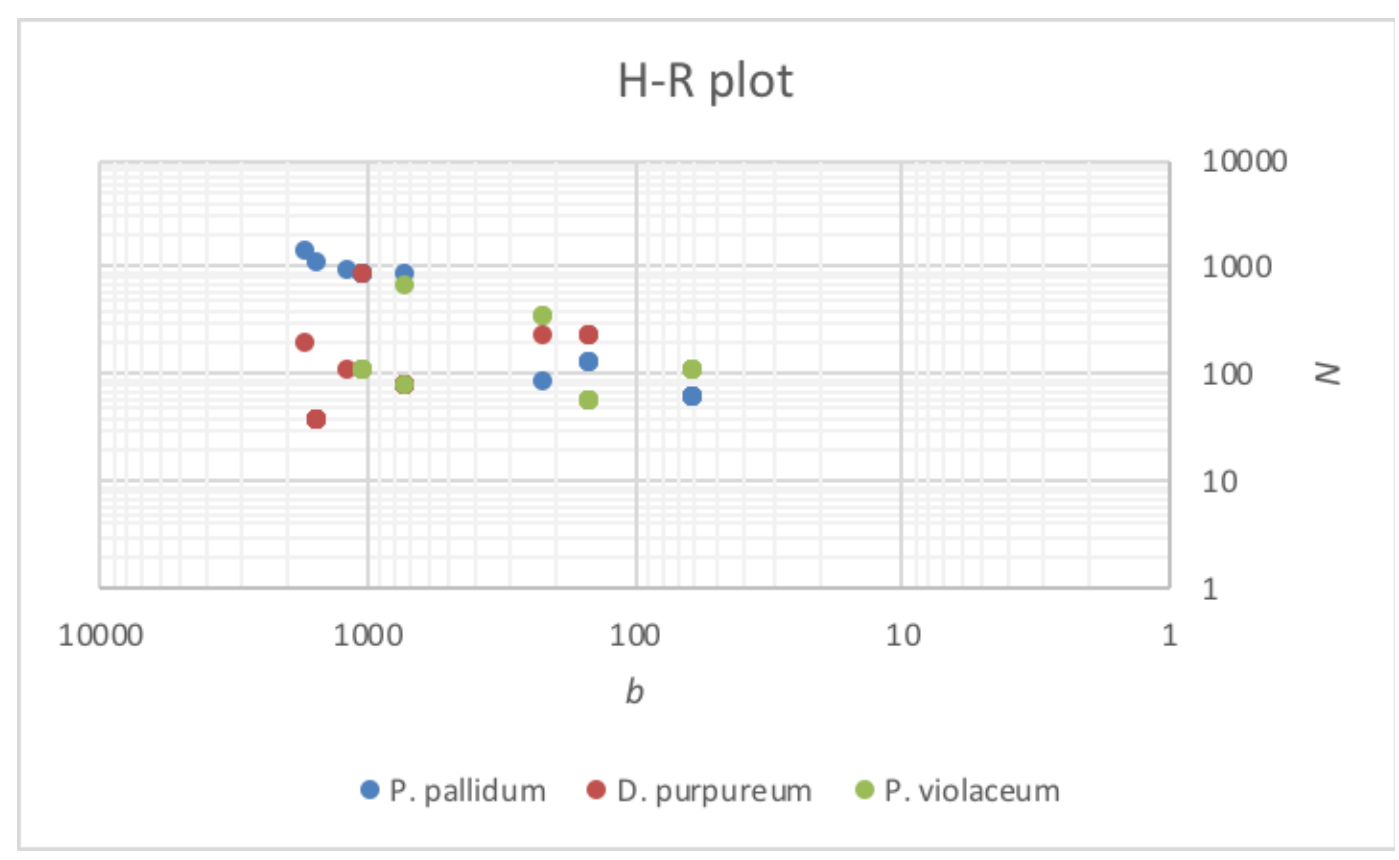

Figure 2: Phase diagram for species. 
a radius of gyration). With an analogue of color as $C=\sqrt{\frac{1}{3}}(R \bar{R}+G \bar{G}+B \bar{B})$, $\exp (C)^{2}=\exp \left(\frac{1}{3}(\Im(s) D)\right)^{3}$ results in $\Im(s) D=(R \bar{R})^{2}+R \bar{R} G \bar{G}+B \bar{B} R \bar{R}$, or a permutation with $R, G, B$, in the sense of a QCD analogue. Color thus becomes valuation of interacting particles. Three-body interaction of $\Re(s)=2$ (hence, with $\Re(s)=-2$ as future) and $\Re(s)=5$ is an example of the case. This $l=5$ case should be regarded as two different aspects. We can consider $\tau_{\rho}=\frac{1}{12}\left(\frac{1}{4}-\rho^{2}\right) \equiv 2\left(1+\rho^{2}\right)(\bmod 5)$ as in [Mochizuki, 1999]. $\rho=0$ results in $\overline{\mathcal{N}}_{1,1}^{0}$ being divided to 2 over $\overline{\mathcal{M}}_{1,1}$ and 3 over $\overline{\mathcal{M}}_{1,1}$; this is an aspect from the same layer as the observant, consisting of $l=2,2,3$ as the three bodies. Similarly, $\rho=1$ results in $\overline{\mathcal{N}}_{1,1}^{1}$ being divided to 1 over $\overline{\mathcal{M}}_{1,1}$ and 3 over $\overline{\mathcal{M}}_{1,1}$ (reduced); this is an aspect from the same layer as the observer, observing $\Re(s)=4 \rightarrow 3$ from 1 . On the other hand, $\rho= \pm i$ results in $\overline{\mathcal{N}}_{1,1}^{-1} \cong \overline{\mathcal{N}}_{1,0}$; this is an aspect from the upper layer from the layer of the observer. In the latter case, the constituents of $l=2+3=5$ can be regarded as forming a solitary acting on the original $l=2$ constituent.

For further clarification, consider D-fivebranes superstring theory [Witten, 1995, Lambert, 1998]. $\mathbb{R}^{4}(3+1)$ case [Adachi, 2019b] would develop one-number ADHM instanton [Witten, 1995], and the potential energy is $V=\frac{1}{8}\left(X^{2}+\right.$ $\left.\rho^{2}\right) \phi^{2}$. Our case implies $(X, \phi)=(1,4)$ and $\rho$ is either 0 with a branch, 1 for normal observation, or $\pm i$ with a fractal. A massless model would result in D-fivebranes $\Re(s)=5$ with type I or type IIA, B superstring theory with $K 3 \times S^{1}$ [Lambert, 1998] $(11-5=6$ dimensions compactified on Mtheory). Since a $K 3$ surface might be related to the $M_{24}$ group as a subgroup [Adachi, 2019a], $M_{24}$ is related to our model in the sense of condensed matter physics described by superstring theory.

This D5 system is analogical to the D1-D5 system in [Martinec et al., 1999]. When $H_{i}=1+\left(\frac{q_{i}}{D}\right)^{2}, i=1,5 ; h=1-\left(\frac{r_{H}}{D}\right)^{2}$, the Lorentz metric is

$$
d s_{M}^{2}=\frac{-h d t^{2}+d D_{5}^{2}}{\sqrt{H_{1} H_{5}}}+\sqrt{\frac{H_{1}}{H_{5}}} d D_{\|}^{2}+\frac{h^{-1} d D^{2}+d \Omega_{3}^{2}}{\sqrt{H_{1} H_{5}}} .
$$

Sending the length of string $l_{s}=2 \pi l \rightarrow 0$ (as observant, [Adachi, 2019a]) would result in

$$
\frac{d s_{M}^{2}}{l_{s}^{2}}=\frac{-h d t^{2}+d D_{5}^{2}}{\sqrt{g_{s}^{2} Q_{1} Q_{5}}}\left(\frac{D}{l_{2}^{2}}\right)^{2}+\sqrt{\frac{Q_{1}}{Q_{5}}}\left[\frac{d D_{\|}^{2}}{V_{4}^{1 / 2}}\right]+\frac{h^{-1}\left(\frac{d D}{D}\right)^{2}+d \Omega_{3}^{2}}{\sqrt{g_{6}^{2} Q_{1} Q_{5}}},
$$

with $Q$ being an instanton and $V_{4}=\Sigma_{1} \Sigma_{2} \Sigma_{3} \Sigma_{4}$. The geometry is locally $A d S_{3} \times S^{3} \times T^{4}$, the radius of $S^{3}$ is $R_{A d S}=l_{s}\left(g_{6}^{2} Q_{1} Q_{5}\right)^{1 / 4}$, and the char- 
498

acteristic proper size of $T^{4}$ is $l_{s}\left(Q_{1} / Q_{5}\right)^{1 / 4}$. This would lead to a harmonic function

$$
H=\sum_{\alpha} \frac{q_{\alpha}^{2}}{\left|D / D_{\alpha}\right|^{2}}, h=0 ;\left(D \sim r_{H}\right)
$$

as the inverse square law when $M_{\alpha}=q_{\alpha}$.

Physical Lagrangian densities for quantum electrodynamics (QED) and QCD are

$$
\mathfrak{L}=\bar{\psi}\left(i c \gamma^{\mu} \partial_{\mu}-M c^{2}\right) \psi+e \bar{\psi} \gamma^{\mu} A_{\mu} \psi-\frac{1}{4 \mu_{0}} F_{\mu \nu} F^{\mu \nu}
$$

$$
\mathfrak{L}=\bar{q}\left(i c \gamma^{\mu} \partial_{\mu}-M c^{2}\right) q+g\left(\bar{q} \gamma^{\mu} T_{a} q\right) G_{\mu}^{a}-\frac{1}{4} G_{\mu \nu}^{a} G_{a}^{\mu \nu} .
$$

In the sense of Lefschetz operator $(i \bar{\partial})$ for multiplication, this is in the form

$$
\mathfrak{L}=\frac{1}{2}\left(\partial_{\mu} \phi_{i}\right)^{2}-\frac{1}{2} \mu^{2} \phi_{i}^{2}-\frac{1}{4} \lambda\left(\phi_{i}\right)^{4}
$$

A counterpart of these in integrated form in our model is

$$
\mathfrak{L}=\frac{1}{2}\left(-s w \Im(s)-M \Im(s)^{2}\right) \phi_{i}^{2}+\frac{1}{2} p^{2} \phi_{i}^{2}-\frac{1}{4} l \frac{\Im(s)}{D}\left(\phi_{i}\right)^{4} .
$$

The first term is diminishing mass, the second is the remaining Frobenioid, and the third is the contribution to fractals. $\phi_{i}$ would be Selberg zeta $\zeta_{\Gamma}$, Hasse-Weil $L$ as in [Adachi, 2019a] or a field $\left|\mathbf{f}_{s}\right|=\frac{\Im(s)}{4 \pi} \frac{M}{D^{3}}(s \rightarrow \Re(s), w \rightarrow$ $\Re(s)-1$ ) as indicated earlier (Tables 6-8). Empirically, $H(t) \approx 0$ and the effect of this parameter is neglected. For a Hamiltonian, they are significantly low when black hole analogies are prominent. For a Lagrangian, negative values may indicate a contribution to fractals composed by climax species Polysphondylium pallidum, while positive values may be anti-fractal species dynamics attributable to pioneering species Dictyostelium purpureum \& Polysphondylium violaceum (refer to [Adachi, 2015]).

The energy

$$
E=\sum_{k} \frac{\partial \mathfrak{L}}{\partial \dot{q}_{k}} \dot{q}-\mathfrak{L}=\mathfrak{H}=\frac{1}{2}\left(-s w \Im(s)-M \Im(s)^{2}\right) \phi_{k}^{2}-\frac{1}{2} p^{2} \phi_{k}^{2}+\frac{1}{4} l \frac{\Im(s)}{D}\left(\phi_{k}\right)^{4},
$$

and

$$
p_{k}=\frac{\partial \mathfrak{L}}{\partial \dot{q}_{k}}=\frac{-s w \Im(s)-M \Im(s)^{2}}{H(t) D} \phi_{k}^{2} .
$$


Table 6: $\left|\mathbf{f}_{s}\right|$ values.

\begin{tabular}{|l|l|l|l|l|l|l|}
\hline$\left|\mathbf{f}_{s}\right|$ & WE P. pallidum & WE D. purpureum & WE P. violaceum & WW P. pallidum & WW D. purpureum & WW P. violaceum \\
\hline May & & & & & & \\
\hline June & 0.2045 & 6.189 & 0.3291 & & & \\
\hline July & & & & 0.3998 & 0.1470 & \\
\hline August & & & & 1.575 & 0.1307 & \\
\hline September & 1.638 & 0.1253 & & 2.211 & 0.4583 & \\
\hline October & 3.484 & 0.09617 & & 1.575 & & 0.1294 \\
\hline November & 0.2209 & & 12.38 & & & \\
\hline December & & & & & & \\
\hline January & & & & & & \\
\hline
\end{tabular}

WE: Washidu East quadrat, WW: Washidu West quadrat; P. pallidum: Polysphondylium pallidum; D. purpureum: Dictyostelium purpureum; P. violaceum: Polysphondylium violaceum. Numbers in red indicate significantly large values deduced by a black hole analogy. Blank cells signify not calculable.

Table 7: Lagrangian.

\begin{tabular}{|c|c|c|c|c|c|c|}
\hline $\mathfrak{L}$ & WE P. pallidum & WE D. purpureum & WE P. violaceum & WW P. pallidum & WW D. purpureum & WW P. violaceum \\
\hline \multicolumn{7}{|l|}{ May } \\
\hline June & -0.3983 & $7.609 \mathrm{E}+5$ & -5.164 & & & \\
\hline July & & & & 5.676 & -0.08279 & $1.907 \mathrm{E}+6$ \\
\hline August & & & & -899.8995563 & -0.800427157 & \\
\hline September & -1072 & -0.9108 & & -2036 & 2485 & \\
\hline October & $-2.368 \mathrm{E}+4$ & -2.932 & & -906.8 & & -0.8065 \\
\hline November & -0.4868 & & $2.820 \mathrm{E}+7$ & & & \\
\hline \multicolumn{7}{|l|}{ December } \\
\hline January & & & & & & \\
\hline
\end{tabular}

WE: Washidu East quadrat, WW: Washidu West quadrat; P. pallidum: Polysphondylium pallidum; D. purpureum: Dictyostelium purpureum; P. violaceum: Polysphondylium violaceum. Numbers in red indicate significantly large absolute values deduced by a black hole analogy. Blank cells signify not calculable. 
Table 8: Hamiltonian.

\begin{tabular}{|l|l|l|l|l|l|l|}
\hline $\mathfrak{H}$ & WE $P$. pallidum & WE $D$. purpureum & WE P. violaceum & WW P. pallidum & WW D. purpureum & WW P. violaceum \\
\hline May & & & & & & \\
\hline June & -0.4346 & $-1.230 \mathrm{E}+6$ & -10.24 & & & \\
\hline July & & & & -39.89 & -0.1037 & $-2.707 \mathrm{E}+6$ \\
\hline August & & & & -684.2 & -0.8629 & \\
\hline September & -798.1 & -0.9680 & -4.487 & -5601 & -2686 & -0.8678 \\
\hline October & $-1.278 \mathrm{E}+4$ & $-3.558 \mathrm{E}+7$ & -690.0 & & \\
\hline November & -0.5288 & & & & & \\
\hline December & & & & & & \\
\hline January & & & & & & \\
\hline
\end{tabular}

WE: Washidu East quadrat, WW: Washidu West quadrat; P. pallidum: Polysphondylium pallidum; D. purpureum: Dictyostelium purpureum; P. violaceum: Polysphondylium violaceum. Numbers in red indicate significantly large absolute values deduced by a black hole analogy. Blank cells signify not calculable.

$\dot{q}_{k}=p_{k} / M$ and

$$
D=\frac{i}{H(t)} \sqrt{\frac{s w \Im(s)}{M}+\Im(s)^{2}} \cdot \phi_{k} .
$$

The sign is for a weak interaction analogue and it completely depends on environmental factors in our biological model. This completes our integrated model for biology analogical to the standard model plus gravity theory, investing a single dimension with a topology [Adachi, 2019b] to a dynamical system with a Lagrangian and Hamiltonian.

\subsection{Further clarification of our model}

If we regard $D=u-w=K$ as an elliptic integral, $f(u)=\sum \frac{1}{D_{i}^{3}}$ has dual periodicity and Weierstraß $\wp=-\zeta^{\prime}(z ; \Lambda)$, where Weierstraß zeta would be $\zeta(z ; \Lambda)=\frac{1}{z}+\sum_{w \in \Lambda^{*}}\left(\frac{1}{z-w}+\frac{1}{w}+\frac{z}{w^{2}}\right)$. Weierstraß zeta accounts for observations from multiple $w$ and 0 accompanied with an absolute zeta function from duplications of $w$. Furthermore, a set of differentials of the first kind of $K$, $\mathfrak{L}_{0} \ni w_{i} d z$, and one-dimensional topologically congruent group $B_{1}^{*}(\mathfrak{R})$ with real coefficients become isomorphic as a $k_{0}$-module. An Abel-Jacobi map shows that

$$
\overline{\mathfrak{D}}_{0}:=\mathfrak{D}_{0} / \mathfrak{D}_{H} \cong V_{g}(k) / P\left(w_{i} d z\right)
$$


$\mathfrak{D}_{0}$ is a 0-dimensional factor group of $K$ and $\mathfrak{D}_{H}$ is a principal factor group of $K$. $D$ could be regarded as a periodic group, $P\left(w_{i} d z\right)$. Thus $\mathfrak{D}_{H} \cong P\left(w_{i} d z\right)$. A $g$-dimensional complex vector module of a modulus $k, V_{g}(k) \cong \mathfrak{D}_{0}$, could be $\operatorname{sign}\left(M_{i}\right) M_{i}\left(M_{i}\right.$ can be $\left.\Im(s)\right)$. Neglecting factorization by a solid angle $4 \pi$, the inverse square law could be multiplication of three $\overline{\mathfrak{D}}_{0}$ in the Abel-Jacobi map.

Interestingly, calculation using physical values from 2018 CODATA shows $\sqrt{k_{g} \sqrt{\varepsilon_{0} \mu_{0}}} \sim 1.9943 \sim 2$, and $\mathbf{F}_{s}=\frac{\operatorname{sign}(M)}{8 \pi} \frac{M_{i} M_{j}}{D^{2}} \mathbf{e}_{\mathbf{F}}$, fulfilling equal partition of forces to outward and inward directions of a particular sphere with radius $D$. This also suggests the branching of the gravity force occurred first, and the branching of the electric (communicative "étale") from the magnetic force $\left(=4 \pi \times 10^{-7}\right.$ and thus a Frobenioid) occurred afterwards. The subtle difference from 2 value indicates neglecting the effect from weak interaction, whose calculation is not straightforward in our model. Even in the standard model, a neutrino is massless and this differs from empirical understandings.

For the SI unit system, the dimension of this value is $\frac{\sqrt{\mathrm{kg} \cdot \mathrm{s}^{3}}}{\mathrm{~m}^{2}}$. In our unit system, it is dimensionless. The constant would be $\frac{\sqrt{\phi} t^{3}}{D^{2}}$. The measure of root of observed $\phi, \sqrt{\phi}$, is equivalent to $t$ (a root of physical time), and the measure of $D$ is a square of $t$, indicating observation linked to interaction. That is, a multiplication of a root of the observant and a cube of $t$ is a duplication of observed fitness of $D^{2}$, indicating duplicated fitness in the potential cube- $t$ space. For further development, considering Einstein's equation

$$
R_{i k}-\frac{1}{2} g_{i k} R=\frac{8 \pi G}{c^{4}} T_{i k}
$$

where $G=1 / 8 \pi, c=\Im(s), T_{i k}=\phi \Im(s)^{2} D^{2} \sqrt{\frac{\Im(s)^{2}+(H(t) D)^{2}}{\Im(s)^{2}-(H(t) D)^{2}}}, \dot{D}=H(t) D$, and the curvature of the left-hand side equals to $\phi \cdot\left(\frac{\dot{D}}{H(t) \Im(s)}\right)^{2} \cdot \sqrt{\frac{\Im(s)^{2}+(H(t) D)^{2}}{\Im(s)^{2}-(H(t) D)^{2}}}$. This means, if the system is abstracted to a sphere, $1 / r^{2}=\phi \cdot\left(\frac{\dot{D}}{H(t) \Im(s)}\right)^{2}$. $\sqrt{\frac{\Im(s)^{2}+(H(t) D)^{2}}{\Im(s)^{2}-(H(t) D)^{2}}}$ and $r=H(t) \Im(s) / \dot{D} \sqrt{\phi \sqrt{\frac{\Im(s)^{2}+(H(t) D)^{2}}{\Im(s)^{2}-(H(t) D)^{2}}}}$. It is also notable that the Ricci curvature tensor is a multiplication of the metric tensor of 1 and the ratio of étale to Frobenioid, i.e., the determinant with matrix from the second fundamental form to that from first fundamental form. The resultant term appears as $\phi \cdot\left(\frac{\dot{D}}{H(t) \Im(s)}\right)^{2} \cdot \sqrt{\frac{\Im(s)^{2}+(H(t) D)^{2}}{\Im(s)^{2}-(H(t) D)^{2}}}$, and $t \rightarrow \infty, \Im(s) \gg H(t) D$ leads to $4 \phi(\dot{D} / \Im(s))^{2}$. 
559

The Macdonald formula shows

$$
\prod_{m=1}^{\infty}\left(1-q^{2 m}\right)^{k}=\sum_{m=0}^{\infty} a_{m, k} q^{m} .
$$

If $\mathfrak{g}=\mathfrak{s l}_{2}$, then $k=3$ and it becomes

$$
\sum_{m=-\infty}^{\infty}(-1)^{m}(2 m+1)\left(q^{m(m+1)}\right)=2 \prod_{m=1}^{\infty}\left(1-q^{2 m}\right)^{3} .
$$

Regarding $D_{m}^{-1}=\left(1-q^{2 m}\right)$, a whole product of infinite $D$ results in multiplication of the value by 2 , similar to the situation above. Regarding bosonic fitness $w, 2 m+1$ means a chaotic situation and this underlies the inverse square law, if the forces are not multiply split.

It is notable that by introducing a squared part for mass, $H(t) D>\Im(s)$ means the mass becomes a purely imaginary number, not a real number. The dimension for the potential as an imaginary axis thus rotates when multiplied by $-i$, and it becomes a real axis. Thus, the potential could be converted to a fractal dimension and it becomes apparent when the velocity of increasing $D$ is high enough such that $H(t) D>\Im(s)$, which is true for zero points of a Riemann zeta function as expected (also refer to [Adachi, 2019a]). When the velocity is approximately $\Im(s)$, the first-order approximation for kinetic energy is $\frac{1}{2} M \Im(s)^{2}$. This is achieved in $E=M \Im(s)^{2}$ energy theory when $(H(t) D)^{2}=5 \Im(s)^{2}$. Thus, for creating fractals beyond $\Re(s)=2$ (square law), one needs recognization from 5-dimensioned fractals in each for $H(t) D \approx \Im(s)$. This is useful for between $\Re(s)=5$ and $\Re(s) \sim 2$ for the development of fractals. The energy related to these forces means energy for creating fractals. For example, recall $s=\frac{\sqrt{7}+i}{2}$ as indicated earlier. The selfinteraction of $s$ results in $\frac{7}{4} e_{\Re(s)}^{2}+\frac{2 \sqrt{7}}{4} i e_{\Re(s)} e_{\Im(s)}-\frac{1}{4} e_{\Im(s)}^{2}$. Thus, the weight for the first term is $\frac{4}{7}$ and it should be an integer. The second term is not an integer and the third term is a multiple of 4 , and neither of them should be considered further. For the Pontryagin class (or, a part of the Hirzebruch signature as indicated in [Adachi, 2019b]), $p_{2}(M)=\frac{4(2 i-1)^{2}+45}{7}$ and the first term corresponds to $p_{2}(M)$ as $\Re(s)=2 i-1\left(p_{1}(M)=2(2 i-1)\right)$. If the condition $i \equiv 0,1 \bmod 7$ is fulfilled, then $p_{2}(M)$ is an integer and $M$ is a differential manifold. However, if not, $M$ is an exotic sphere that is homeomorphic but not diffeomorphic to the standard Euclidean $S^{7}$. For a continuous time model by differentials, this is not allowed and development 
into $\Re(s) \geq 5$ should be achieved in partners of $\Re\left(s_{1}\right)+\Re\left(s_{2}\right)=7 j, 7 j+1, j \in$ $\mathbb{N}$ (e.g., $\Re(s)=5$ and $\Re(s)=2$ observed in October at the Washidu East quadrat between Polysphondylium pallidum and Dictyostelium purpureum [Adachi, 2019a]).

\subsection{Roles of another analogy to quantum mechanics in our model}

If we set $\phi\left|\alpha_{S}(t)\right\rangle=l\left|\alpha_{S}(t)\right\rangle$ and $\left\langle\alpha_{S}(t)\right| \phi=\left\langle\alpha_{S}(t)\right| p$, then the bra becomes the basic bra and the ket becomes the standard ket. The former represents phase information (Frobenioid; persistence homology), and the latter represents the norm (étale function or cohomology). Note that $l$ is suitable for communication because of its characteristics beyond the restricted layer of a particular system. Setting the $\Phi(\alpha)=0$ function as a monic polynomial for a cyclotomic field, the polynomial becomes a zero ideal of the ring structure for the bra or ket. The eigenvalues for $|\alpha(t)\rangle\langle\alpha(t)|$ are either 1 or 0 (an observed state or non-observed state by the interactions between a particular set of kets and bras), and this information is usable for Morse code for the communication. Their integration becomes a Dirac $\delta$ function, that is, $\Re(s)=l$. Only where $p=l,\langle\alpha(t)|\phi| \alpha(t)\rangle$ leads to $E(N)$. For the $H=H_{0}+H^{\prime}$ case of $M_{C}$ and $M_{W}, H_{0}=l, H^{\prime}=-l$, and $H=l+(-l)=0$. This means $M_{W}$ is invested with a fractal structure of $l$-dimensions, while $M_{C}$ loses the same number of fractal dimensions. The time development will result in a static state of $M_{W}$ being invested with everything if the relation continues at all.

The entanglement entropy of these two harmonic oscillators is

$$
S_{A}=\cosh ^{2} \theta \ln \cosh ^{2} \theta-\sinh ^{2} \theta \ln \sinh ^{2} \theta
$$

where $\theta$ is expressed by $l=\frac{2 \sinh \theta \cosh \theta}{1+2 \sinh ^{2} \theta}$ with Hamiltonian $H=w^{\dagger} w+$ $c^{\dagger} c+l\left(w^{\dagger} c^{\dagger}+w c\right)$, when $\left(w, w^{\dagger}\right),\left(c, c^{\dagger}\right)$ are creation-annihilation operators of $M_{W}, M_{C}$. For maximum entanglement, consider Hilbert spaces $\mathcal{H}_{W}, \mathcal{H}_{C}$. The dimensions of the spaces are $\left|\mathcal{H}_{W}\right|=\sum N_{W},\left|\mathcal{H}_{C}\right|=\sum N_{C}$ and $\left.\min \left(\ln \left|\mathcal{H}_{W}\right|, \ln \left|\mathcal{H}_{C}\right|\right)\right)$ is their maximum entanglement entropy. If $M_{W}$ absorbs all of $N_{k}$ in $M_{C}$, then $\ln \left|\mathcal{H}_{C}\right|=\ln P+N_{k} \frac{s}{b}$ and the second term approaches zero. Thus, $\ln P$ is the entanglement entropy attributable to diminishing $N_{k}$.

It is notable that once assuming an analogy to QED of the Frobenioidétale relationships, at least for $\Re(s)=2,3$, hetero-interaction terms become negligible according to QED, which accords well with empirical results 
[Adachi, 2019a]. For the most efficient communication that communicates all the étales and Frobenioids, as both of them are in parallel, the following condition is needed:

$$
\frac{\frac{\mathbf{v}}{\Im(s)}}{1+\frac{\mathbf{V}^{2}}{\Im(s)^{2}}}=\frac{\mathbf{E} \times \mathbf{H}}{E^{2}+H^{2}}
$$

[Landau and Lifshitz, 1975]. Along this line, a conservation law in $D$-space could be

$$
\frac{\partial}{\partial t}\left\{\frac{E^{2}+H^{2}}{2} d V+\sum \mathscr{E}_{\text {kin }}\right\}=-\oint \mathbf{S} d \mathbf{f}(=0),
$$

where a Poynting vector $\mathbf{S}=\Im(s) \mathbf{E} \times \mathbf{H}$ and $d \mathbf{f}$ is an areal element. $(=0)$ is achieved when the volume is infinite.

\subsection{From renormalization to fractals}

Furthermore, in our biological model, we can attribute hierarchical formation to the solution for the divergence problem of transition probability, as of renormalization. Terms of harmonic oscillators for a Hamiltonian of QED become divergent unless renormalized. However, we can treat each of the oscillators as an additional dimension of a fractal structure. Mathematically, the plausibility of renormalization as $P^{m}$ would be defined as the existence of $0 \in U,\left(P^{m}, U, V\right)$ being a mapping similar to a quadratic and a filled Julia set $K_{m}$ being connected, when the quadratic $P(z)=z^{2}+c$ and $U, V$ are simply connected regions. The condition is fulfilled in a continuously developing system constituted by components of simply connected regions with an observant $U$ in our case. The renormalization equation would be

$$
\mu \frac{d \mathfrak{M}}{d \mu}=\left(\left.\mu \frac{\partial}{\partial \mu}\right|_{e}+\mu \frac{\partial e}{\partial \mu} \frac{\partial}{\partial e}\right) \mathfrak{M}=0 .
$$

The first and second terms on the left-hand side are renormalizing and remaining terms, respectively. These have negative and positive values, and represent the contribution of $\mathfrak{M}$ to fractal structure. Or, we may state that a fractal $l$-dimension is for one of the periods of an elliptic function that acts on a "string".

However, the plausibility of renormalization is restricted to $d<8$, at most, in the $\mathbb{R}^{3} \times \mathbb{R}^{3} \cong \hat{\mathbb{C}}$ case. For further application, a similar statement is true for the Bogomolov conjecture. Let $A$ be an abelian manifold on 
an algebraic field $K$. Let $L$ be a symmetric and enriched invertible sheaf on $A$ and $A_{\bar{K}}:=A \times_{\operatorname{Spec}(K)} \operatorname{Spec}(\bar{K}) . X$ would be an irreducible algebraic submanifold of $A_{\bar{K}}$. When $\hat{h}_{L}$ is a Néron-Tate height function on $L$ and $\forall \varepsilon>0$, a set $\left\{x \in X(\bar{K}) \mid \hat{h}_{L}(x) \leq \varepsilon\right\}$ is dense in the sense of Zariski topology in $X$, there exists a subset of an abelian manifold $B$, and a torsion point $b$ on $A_{\bar{K}}$ s.t. $X=B+b$. Regarding $b$ as analogical to the term for renormalization, one can simply consider $B$ for the current layer and $b$ is a partial point of fractals, or a fractal string. That is, $B$ is a Frobenioid and $b$ is an étale.

\section{Discussion}

As per [Mochizuki, 2019d], a species is roughly a collection of set-theoretic formulas that gives rise to a category in any given model of set theory. For example, we can define them as a $k$-set of a single dimension $N_{k}$ with a certain topology [Adachi, 2019b], or a set of $(p, l, v)$ with particular morphisms. This paper in conjunction with [Adachi, 2019a, Adachi, 2019b] complete our integrated model of species dynamics. Various interpretations according to alternative mathematical theorems are as follows.

If $\varnothing \neq \partial B$, then the Poisson-Jensen formula is applicable to $D=e^{s / b}$ [Adachi, 2019a] and

$$
\Re(s)=-b \sum_{i=1}^{n} \ln \left|\frac{\rho^{2}-\bar{a}_{i} z}{\rho\left(z-a_{i}\right)}\right|+\frac{1}{2 \pi} \int_{0}^{2 \pi} \Re\left(\frac{\rho e^{i \theta}+z}{\rho e^{i \theta}-z}\right) \rho d \theta,
$$

where $a_{i}$ is a zero point of $w=D$ [Adachi, 2019b]. The first term should be the contributions from the discrete part of $\Re(s)$ depending on the zero points of $w$ (observation phenomena) and the second term should be that from the continuous part of $\Re(s)(0 \leq \Re(s)<2)$ [Adachi, 2019a, Adachi, 2019b].

In another expression, we can regard this equation as a unique Lebesgue decomposition in the Radon-Nikodym theorem:

$$
\Phi(E)=F(E)+\Psi(E),
$$

where $s \in E$, a set function $\Phi(E)$ has a sigma additivity on $B$, a set function $F(E)$ has an absolute continuity, and a set function $\Psi(E)$ has singulars. Since $F(E)=\Im(s), \Psi(E)=\Re(s)$, and they are expressed by the Riemann-Hurwitz formula in [Adachi, 2019a], $\Phi(E)$ can uniquely determine the decomposition 
of contributions from the same hierarchy of interest $(F(E))$ and other hierarchies above $(\Psi(E))$. For another expression, universal coefficient theorems [Bott and $\mathrm{Tu}, 1982$ ] show (a) the homology of $B$ with an abelian group of a $B$-module $G$,

$$
H_{q}(B ; G) \cong H_{q}(B) \otimes G \oplus \operatorname{Tor}\left(H_{q-1}(B), G\right)
$$

where the first term is exactly $\Im(s)$ and the second term is $\Re(s)$ in [Adachi, 2019a];

(b) the cohomology of $B$ with an abelian group of a $B$-module $G$,

$$
H^{q}(B ; G) \cong \operatorname{Hom}\left(H_{q}(B), G\right) \oplus \operatorname{Ext}\left(H_{q-1}(B), G\right),
$$

where the first term is exactly $\Im(s)$ and the second term is $\Re(s)$ in [Adachi, 2019a].

Note that, by Hodge's theorem, the cohomology is isomorphic to harmonic oscillators in the sense that the numbers of dimensions of the former and the latter are equivalent. The cohomological dimension thus represents the number of harmonic oscillators underlying in the system of interest.

All of these mathematical formulas have interpretive value in terms of particular aspects of renormalization or fractals acting on multilevel layers and, as in biology, multilevel selections. We emphasize the potential of divergent terms as acting on higher-order hierarchies such as fractals.

\section{Acknowledgments}

I am very grateful to reviewers and colleagues for their comments and advice spanning biology, mathematics, and physics.

\section{References}

[Adachi, 2015] Adachi, S.: Eastern Japanese Dictyostelia species adapt while populations exhibit neutrality. Evol. Biol. 42 210-222 (2015). https://doi.org/10.1007/s11692-015-9312-0.

[Adachi, 2017] Adachi, S.: Rigid geometry solves “curse of dimensionality" effects in clustering methods: An application to omics data. PLOS ONE 12, e0179180 (2017). https://doi.org/10.1371/journal.pone.0179180. 
[Adachi, 2019a] Adachi, S.: Exploring group theory and topology for analyzing the structure of biological hierarchies, arXiv:1603.00959v8 [qbio.PE] (2019). https://arxiv.org/abs/1603.00959.

[Adachi, 2019b] Adachi, S.: Induction of hierarchy and time through one-dimensional probability space with certain topologies, bioRxiv:780882v2 (2019). https://www.biorxiv.org/content/10.1101/780882v2.

[Ahlfors, 1979] Ahlfors, L. V.: Complex Analysis. McGraw-Hill Book Company, New York (1979)

[Benfatto and Gallavotti, 1995] Benfatto, G., Gallavotti, G.: Renormalization Group. Princeton University Press, Princeton (1995)

[Borcherds, 1996] Borcherds, R. E.: Automorphic forms and Lie algebras. Current Developments in Mathematics 1996, 1-36 (1996). http://dx.doi.org/10.4310/CDM.1996.v1996.n1.a1.

[Bott and Tu, 1982] Bott, R. B., Tu, L. W.: Differential Forms in Algebraic Topology. Springer-Verlag, New York (1982)

[Cardy, 1996] Cardy, J.: Scaling and Renormalization in Statistical Physics. Cambridge University Press, Cambridge (1996)

[Emch, 1899-1900] Emch, A.: Illustration of the elliptic integrals of the first kind by a certain link-work. Ann. Math. 2nd Ser. 1 1899-1900, 81-92 (1899-1900). http://doi.org/10.2307/1967273.

[Emch, 1900-1901] Emch, A.: An application of elliptic functions to Peaucellier's link-work (inversor). Ann. Math. 2nd Ser. 2 1900-1901, 60-63 (1900-1901). http://doi.org/10.2307/2007182.

[Hartshorne, 1977] Hartshorne, R.: Algebraic Geometry. Springer, New York (1977)

[Kachru and Tripathy, 2017] Kachru, S., Tripathy, A.: The hidden symmetry of the heterotic string. Adv. Theor. Math. Phys. 21, 1729-1745 (2017). https://dx.doi.org/10.4310/ATMP.2017.v21.n7.a5. 
[Kashiwara and Schapira, 2018] Kashiwara, M., Schapira, P.: Persistent homology and microlocal sheaf theory. J. Appl. Coput. Topol. 2, 83-113 (2018). https://doi.org/10.1007/s41468-018-0019-z.

[Kedlaya, 2010] Kedlaya, K. S.: p-adic Differential Equations. Cambridge University press, New York (2010)

[Kramár, 2016] Kramár, M., Levanger, R., Tithof, J., Suri, B., Xu, M., Paul, M., et al.: Analysis of Kolmogorov flow and Rayleigh-Bénard convection using persistent homology. Physica D 334, 82-98 (2016). https://doi.org/10.1016/j.physd.2016.02.003.

[Lambert, 1998] Lambert, N. D.: D-brane bound states and the generalised ADHM construction. Nucl. Phys. B 519, 214-224 (1998). https://doi.org/10.1016/S0550-3213(98)00026-1.

[Landau and Lifshitz, 1975] Landau, L.D., Lifshitz, E. M.: The Classical Theory of Fields, Vol. 2, 4th ed. Butterworth-Heinemann, Oxford (1975)

[Martinec et al., 1999] Martinec, E. J., Nozaki, M., Takayanagi, T.: Manifestations of the D1-D5 system. Recent Advances in String Theory (1999). Available from: http://home.catv.ne.jp/pp/takayana/martinec.ps.gz. Accessed 12 October 2019.

[Medlin et al., 1988] Medlin, L., Elwood, H.J.,Stickel, S., Sogin, M.L.: The characterization of enzymatically amplified eukaryotic 16S-like rRNAcoding regions. Gene 71 491-499 (1988). https://doi.org/10.1016/03781119(88)90066-2.

[Mochizuki, 1999] Mochizuki, S.: Foundations of $p$-adic Teichmüller Theory. American Mathematical Society and International Press, Cambridge (1999)

[Mochizuki, 2019a] Mochizuki, S.: $\quad$ Inter-universal Teichmüller Theory I: Construction of Hodge Theaters. Available from: $\quad$ http://www.kurims.kyoto-u.ac.jp/ $\quad$ motizuki/Interuniversal\%20Teichmuller\%20Theory\%20I.pdf. Accessed 18 October 2019. 
[Mochizuki, 2019b] Mochizuki, S.: Inter-universal Teichmüller Theory II: Hodge-Arakelov-theoretic Evaluation. Available from: http://www.kurims.kyoto-u.ac.jp/ $\quad$ motizuki/Interuniversal\%20Teichmuller\%20Theory\%20II.pdf. Accessed 18 October 2019.

[Mochizuki, 2019c] Mochizuki, S.: Inter-universal Teichmüller Theory III: Canonical Splittings of the Log-theta-lattice. Available from: http://www.kurims.kyoto-u.ac.jp/ ${ }^{\sim}$ motizuki/Interuniversal\%20Teichmuller\%20Theory\%20III.pdf. Accessed 18 October 2019 .

[Mochizuki, 2019d] Mochizuki, S.: Inter-universal Teichmüller Theory IV: Log-volume Computations and Set-theoretic Foundations. Available from: http://www.kurims.kyoto-u.ac.jp/ ${ }^{\sim}$ motizuki/Interuniversal\%20Teichmuller\%20Theory\%20IV.pdf. Accessed 18 October 2019.

[Sato, 1959] Sato, M.: Theory of hyperfunctions, I., J. Fac. Sci. Univ. Tokyo, Sect., I. 8, 139-193 (1959)

[Sato, 1960] Sato, M.: Theory of hyperfunctions, II., J. Fac. Sci. Univ. Tokyo, Sect., I. 8, 387-437 (1960).

[Schwartz, 1966] Schwartz, L.: Théorie des Distributions, Hermann \& Cie, Paris (1966)

[Stone, 1930] Stone, M. H.: Linear transformations in Hilbert space: III. Operational methods and group theory. Proc. Natl. Acad. Sci. USA 16, 172-175 (1930). https://doi.org/10.1073/pnas.16.2.172.

[Stone, 1932] Stone, M. H.: On one-parameter unitary groups in Hilbert space. Ann. Math. 33, 643-648 (1932). https://doi.org/10.2307/1968538.

[von Neumann, 1931] von Neumann, J.: Die Eindeutigkeit der Schrödingerschen Operatoren. Math. Ann. 104, 570-578 (1931). https://doi.org/10.1007/BF01457956. 
793

794

795

796

797

798

799

800

801

[von Neumann, 1932] von Neumann, J.: Ueber Einen Satz von Herrn M. H. Stone. Ann. Math. Ser. 2 33, 567-573 (1932). https://doi.org/10.2307/1968535.

[Witten, 1995] Witten, E.: Sigma models and the ADHM construction of instantons. J. Geom. Phys. 15, 215-226 (1995). https://doi.org/10.1016/0393-0440(94)00047-8.

[Witten, 2016] Witten, E.: Integrable lattice models from gauge theory. Adv. Theor. Math. Phys. 21, 1819-1843 (2017). https://dx.doi.org/10.4310/ATMP.2017.v21.n7.a10. 